\title{
Isotropic realizability of fields and reconstruction of invariant measures under positivity properties. Asymptotics of the flow by a non-ergodic approach
}

\author{
Marc Briane \\ Univ Rennes, INSA Rennes, CNRS, IRMAR - UMR 6625, F-35000 Rennes, France \\ mbriane@insa-rennes.fr
}

January 29, 2019

\begin{abstract}
The paper is devoted to the isotropic realizability of a regular gradient field $\nabla u$ or a more general vector field $b$, namely the existence of a continuous positive function $\sigma$ such that $\sigma b$ is divergence free in $\mathbb{R}^{d}$ or in an open set of $\mathbb{R}^{d}$. First, we prove that under some suitable positivity condition satisfied by $\nabla u$, the isotropic realizability of $\nabla u$ holds either in $\mathbb{R}^{d}$ if $\nabla u$ does not vanish, or in the open sets $\left\{c_{j}<u<c_{j+1}\right\}$ if the $c_{j}$ are the critical values of $u$ (including $\inf _{\mathbb{R}^{d}} u$ and $\sup _{\mathbb{R}^{d}} u$ ) which are assumed to be in finite number. It turns out that this positivity condition is not sufficient to ensure the existence of a continuous positive invariant measure $\sigma$ on the torus when $\nabla u$ is periodic. Then, we establish a new criterium of the existence of an invariant measure for the flow associated with a regular periodic vector field $b$, which is based on the equality $b \cdot \nabla v=1$ in $\mathbb{R}^{d}$. We show that this gradient invertibility is not related to the classical ergodic assumption, but it actually appears as an alternative to get the asymptotics of the flow.
\end{abstract}

Keywords: Isotropic realizability, dynamical system, invariant measure, asymptotics of the flow

Mathematics Subject Classification: 37C10, 78A30, 35B27

\section{Introduction}

In this paper we study the problem of the isotropic realizability of a vector field $b \in C^{1}\left(\mathbb{R}^{d}\right)^{d}$, namely the existence of a positive function $\sigma \in C^{0}\left(\mathbb{R}^{d}\right)$ solution to the equation

$$
\operatorname{div}(\sigma b)=0 \text { in } \mathbb{R}^{d},
$$

or in an open subset of $\mathbb{R}^{d}$. When the vector field $b$ is periodic with respect to $Y_{d}:=[0,1)^{d}$, i.e.

$$
\forall \kappa \in \mathbb{Z}^{d}, \forall x \in \mathbb{R}^{d}, \quad b(x+\kappa)=b(x),
$$

the problem of the isotropic realizability in the torus $\mathbb{R}^{d} / \mathbb{Z}^{d}$, namely the existence of a positive $Y_{d}$-periodic function $\sigma \in C^{0}\left(\mathbb{R}^{d}\right)$ solution to (1.1), is also addressed. 
In the case where $b=\nabla u$ is a gradient field, the reconstruction of a positive $\sigma$ has been first done in [2] and a rigorous way in [13] assuming that $\nabla u$ never vanishes and using the method of characteristics. Alternatively, when the potential $u$ satisfies a prescribed boundary condition on a bounded smooth domain of $\mathbb{R}^{2}$ with a finite number of critical points, a conductivity $\sigma$ has been derived in [1] thanks to an approximation procedure adding a vanishing viscosity term. More recently, the isotropic realizability of a non-vanishing gradient, i.e.

$$
\inf _{\mathbb{R}^{d}}|\nabla u|>0,
$$

has been revisited in [6] both in the space $\mathbb{R}^{d}$ and in the torus $\mathbb{R}^{d} / \mathbb{Z}^{d}$ using specifically the flow

$$
\left\{\begin{array}{l}
X^{\prime}(t, x)=b(X(t, x)), \quad t \in \mathbb{R} \\
X(0, x)=x \in \mathbb{R}^{d},
\end{array}\right.
$$

with $b=\nabla u$. In particular, it was proved that the isotropic realizability in the torus is actually stronger that the realizability in the space. Furthermore, again using the flow (1.3) we showed in [4, Theorem 4.1] that in any dimension the presence of critical points for the potential $u$ may be an obstacle to the (even local) existence of a conductivity $\sigma$ solution to (1.1) with $b=\nabla u$. The case of non-regular gradients has been also investigated in [5].

Beyond the negative results of [4] when the non-vanishing condition (1.2) does not hold, we thus need extra conditions on the gradient $\nabla u$ to ensure its isotropic realizability in the whole space $\mathbb{R}^{d}$ or at least in a subset of $\mathbb{R}^{d}$. First, we prove (see Theorem 2.1) that if $u \in C^{2}\left(\mathbb{R}^{d}\right)$ has either all its non-negative partial derivatives or all its non-positive partial derivatives the ratios of which are controlled from above and below (see more precisely condition (2.3) below), and if $u$ has exactly $n$ critical values:

$$
c_{0}:=\inf _{\mathbb{R}^{d}} u<c_{1}=u\left(\xi^{1}\right)<\cdots<c_{n}=u\left(\xi^{n}\right)<c_{n+1}:=\sup _{\mathbb{R}^{d}} u, \quad \text { with } \nabla u\left(\xi^{j}\right)=0,
$$

possibly with $\inf _{\mathbb{R}^{d}}|\nabla u|=0$ (so that $\nabla u$ may vanish at infinity), then $\nabla u$ is isotropically realizable:

- either in $\mathbb{R}^{d}$ when $u$ has no critical point,

- or in the $(n+1)$ open sets $\left\{c_{j}<u<c_{j+1}\right\}$ for $j=0, \ldots, n$.

Then, we extend this result to the isotropic realizability of a vector field $b \in C^{1}\left(\mathbb{R}^{d}\right)^{d}$. Assuming the existence of an open interval $I \subset \mathbb{R}$ and a function $u \in C^{1}\left(\mathbb{R}^{d}\right)$ such that for any $x$ in the inverse image $\{u \in I\}$, the function $u(X(\cdot, x))$ is increasing and its range contains $I$, we show (see Theorem 2.3) the isotropic realizability of $b$ in the open set $\{u \in I\}$.

The isotropic realizability in the torus $\mathbb{R}^{d} / \mathbb{Z}^{d}$ of a $Y_{d}$-periodic vector field $b \in C^{1}\left(\mathbb{R}^{d}\right)^{d}$ is more intricate. In this case by the uniqueness of the Cauchy-Lipschitz theorem the flow $X$ solution to (1.3) satisfies

$$
\forall \kappa \in \mathbb{Z}^{d}, \forall(t, x) \in \mathbb{R} \times \mathbb{R}^{d}, \quad X(t, x+\kappa)=X(t, x)+\kappa,
$$

so that the image of $X(t, x)$ by the canonical surjection $\Pi: \mathbb{R}^{d} \rightarrow \mathbb{R}^{d} / \mathbb{Z}^{d}$ is independent of any representative $x$ in the class $\Pi(x)$. Hence, equation (1.3) is well posed in the torus. Then, by virtue of Liouville theorem (see, e.g., [7, Chap. 2, Theorem 1]) the isotropic realizability (1.1) 
in the torus is equivalent to the existence of a positive $Y_{d}$-periodic function $\sigma \in C^{0}\left(\mathbb{R}^{d}\right)$ which is called an invariant measure for the flow $X$, such that for any $Y_{d}$-periodic function $\varphi \in C^{1}\left(\mathbb{R}^{d}\right)$,

$$
\forall t \in \mathbb{R}, \quad \int_{Y_{d}} \varphi(X(t, x)) \sigma(x) d x=\int_{Y_{d}} \varphi(x) \sigma(x) d x
$$

Furthermore, the natural extension to any vector field $b$ of the condition (2.3) relating to a gradient field, is the boundedness from below by a positive constant of the coefficients $b_{k}$ for $k=1, \ldots, d$, either the coefficients $-b_{k}$. However, it turns out that this boundedness condition is not sufficient to get the existence of an invariant measure as show Proposition 3.1 and Example 3.2.

Actually, assuming that the $Y_{d^{-}}$periodic vector field $b \in C^{1}\left(\mathbb{R}^{d}\right)^{d}$ satisfies the gradient invertibility

$$
b \cdot \nabla v_{1}=1 \text { in } \mathbb{R}^{d}
$$

for some $Y_{d}$-periodic gradient $\nabla v_{1} \in C^{0}\left(\mathbb{R}^{d}\right)^{d}$, we prove (see Theorem 3.3) that the existence of an invariant measure (1.4) for the flow $X$ is equivalent to the existence of a vector $\xi \in \mathbb{R}^{d}$ and $d$ linearly independent $Y_{d}$-periodic gradients $\nabla w_{k} \in C^{0}\left(\mathbb{R}^{d}\right)^{d}$ such that

$$
b \cdot \nabla w_{k}=\xi_{k} \text { in } \mathbb{R}^{d}, \quad \text { for } k=1, \ldots, d .
$$

At this point, we need to replace in dimension $d \geq 3$ the equation (1.1) by the more restrictive condition that $b$ is proportional to a cross product of $(d-1)$ gradients. As a by-product, under condition (1.5) the former equivalence shows (see Corollary 4.1) that the existence of an invariant measure for $X$ implies the asymptotics

$$
\lim _{|t| \rightarrow \infty} \frac{X(t, x)}{t}=\xi \quad \text { for any } x \in \mathbb{R}^{d}
$$

and not only almost everywhere in $\mathbb{R}^{d}$ as obtained by the Birkhoff ergodic theorem. Surprisingly, although the limit $\xi$ is constant, it appears (see Example 4.3) that the flow $X$ is not in general ergodic in dimension $d \geq 2$. Indeed, we may construct a non-constant $Y_{d}$-periodic function which is invariant by the flow $X$. Therefore, it seems that the gradient invertibility (1.5) can be regarded as a substitute for the classical ergodic assumption (see Remark 4.2). This allows us to recover some of the two-dimensional ergodicity results of [16, 11] by a new and nonergodic approach, and to extend partially them to higher dimension. As a natural extension of Corollary 4.1 the homogenization of a linear transport equation with oscillating coefficients (see Corollary 4.4) is derived by the non-ergodic approach. Condition (1.5) in any dimension still plays the same role as the irrationality of the so-called rotation number (see Remark 4.23 .) in the two-dimensional homogenization results of $[3,10,16]$ which are based on the ergodicity of the flow.

\section{Notations}

- $\left(e_{1}, \ldots, e_{d}\right)$ denotes the canonical basis of $\mathbb{R}^{d}$.

- denotes the scalar product in $\mathbb{R}^{d}$.

- $I_{d}$ denotes the unit matrix of $\mathbb{R}^{d \times d}$, and $R_{\perp}$ denotes the clockwise $90^{\circ}$ rotation matrix in $\mathbb{R}^{2 \times 2}$.

- For $M \in \mathbb{R}^{d \times d}, M^{T}$ denotes the transpose of $M$. 
- $Y_{d}:=[0,1)^{d}$, and $\langle f\rangle$ denotes the average-value of a function $f \in L^{1}\left(Y_{d}\right)$.

- $|A|$ denotes the Lebesgue measure of a measurable subset $A$ of $\mathbb{R}^{d}$.

- $C_{\sharp}^{k}\left(Y_{d}\right)$ denotes the space of the $Y_{d}$-periodic functions of class $C^{k}$ in $\mathbb{R}^{d}$.

- $L_{\sharp}^{p}\left(Y_{d}\right), p \geq 1$, denotes the space of the $Y_{d}$-periodic functions in $L_{\mathrm{loc}}^{p}\left(\mathbb{R}^{d}\right)$, and $H_{\sharp}^{1}\left(Y_{d}\right)$ denotes the space of the functions $\varphi \in L_{\sharp}^{2}\left(Y_{d}\right)$ such that $\nabla \varphi \in L_{\sharp}^{2}\left(Y_{d}\right)^{d}$.

- For any open set $\Omega$ of $\mathbb{R}^{d}, C_{c}^{\infty}(\Omega)$ denotes the space of the smooth functions with compact support in $\Omega$.

- For $u \in L_{\mathrm{loc}}^{1}\left(\mathbb{R}^{d}\right)$ and $U=\left(U_{j}\right)_{1 \leq j \leq d} \in L_{\mathrm{loc}}^{1}\left(\mathbb{R}^{d}\right)^{d}$,

$$
\nabla u:=\left(\partial_{x_{1}}, \ldots, \partial_{x_{d}}\right) \quad \text { and } \quad D U:=\left[\partial_{x_{i}} U_{j}\right]_{1 \leq i, j \leq d} .
$$

- For $\xi_{1}^{1}, \ldots, \xi^{d}$ in $\mathbb{R}^{d}$, the cross product $\xi^{2} \times \cdots \times \xi^{d}$ is defined by

$$
\xi^{1} \cdot\left(\xi^{2} \times \cdots \times \xi^{d}\right)=\operatorname{det}\left(\xi^{1}, \xi^{2}, \ldots, \xi^{d}\right) \quad \text { for } \xi^{1} \in \mathbb{R}^{d},
$$

where det is the determinant with respect to the canonical basis $\left(e_{1}, \ldots, e_{d}\right)$, or equivalently, the $k^{\text {th }}$ coordinate of the cross product is given by the $(d-1) \times(d-1)$ determinant

$$
\left(\xi^{2} \times \cdots \times \xi^{d}\right) \cdot e_{k}=(-1)^{k+1}\left|\begin{array}{ccc}
\xi_{1}^{2} & \cdots & \xi_{1}^{d} \\
\vdots & \ddots & \vdots \\
\xi_{k-1}^{2} & \cdots & \xi_{k-1}^{d} \\
\xi_{k+1}^{2} & \cdots & \xi_{k+1}^{d} \\
\vdots & \ddots & \vdots \\
\xi_{d}^{2} & \cdots & \xi_{d}^{d}
\end{array}\right| .
$$

\section{Isotropic realizability of a vector field in $\mathbb{R}^{d}$ under pos- itivity properties}

\subsection{Isotropic realizability of a gradient in $\mathbb{R}^{d}$}

Let $u \in C^{1}\left(\mathbb{R}^{d}\right)$. In this section we assume that the gradient field $b=\nabla u$ has the following positivity properties:

$$
\forall k \in\{1, \ldots, d\}, \quad \partial_{x_{k}} u \geq 0 \text { in } \mathbb{R}^{d} \text { or } \forall k \in\{1, \ldots, d\}, \quad \partial_{x_{k}} u \leq 0 \text { in } \mathbb{R}^{d},
$$

and there exist positive fonctions $\alpha_{k}, \beta_{k} \in C^{0}(\mathbb{R})$ with

$$
\int_{0}^{ \pm \infty} \alpha_{k}(t) d t=\int_{0}^{ \pm \infty} \beta_{k}(t) d t= \pm \infty
$$

such that for any $x \in \mathbb{R}^{d}$, up to renumber the coordinates $x_{k}$,

$$
\forall k \in\{1, \ldots, d-1\}, \quad \frac{\alpha_{k}\left(x_{k}\right)}{\alpha_{k+1}\left(x_{k+1}\right)}\left|\partial_{x_{k}} u(x)\right| \leq\left|\partial_{x_{k+1}} u(x)\right| \leq\left|\partial_{x_{k}} u(x)\right| \frac{\beta_{k}\left(x_{k}\right)}{\beta_{k+1}\left(x_{k+1}\right)} .
$$

Note that in (2.3) the partial derivatives of $u$ may vanish but the ratios between two consecutive partial derivatives are controlled.

We have the following result. 
Theorem 2.1. Let $u \in C^{2}\left(\mathbb{R}^{d}\right)$ be a function satisfying conditions (2.1) and (2.3).

i) Assume that $u$ has no critical point in $\mathbb{R}^{d}$, i.e. $\nabla u$ does not vanish in $\mathbb{R}^{d}$. Then, $\nabla u$ is isotropically realizable in $\mathbb{R}^{d}$ with a positive function $\sigma \in C^{1}\left(\mathbb{R}^{d}\right)$.

ii) Assume that $u$ has a unique critical point $x^{0}$, i.e. $\nabla u\left(x^{0}\right)=0$ and $\nabla u$ does not vanish in $\mathbb{R}^{d} \backslash\left\{x^{0}\right\}$. Then, $\nabla u$ is isotropically realizable with a positive $C^{1}$-function $\sigma$ in the open sets $\left\{u>u\left(x^{0}\right)\right\}$ and $\left\{u<u\left(x^{0}\right)\right\}$.

iii) More generally, assume that there exists a positive integer $n$ such that

$$
\begin{gathered}
u\left(\left\{x \in \mathbb{R}^{d}: \nabla u(x)=0\right\}\right)=\left\{c_{1}, \ldots, c_{n}\right\} \\
\text { with } \inf _{\mathbb{R}^{d}} u=: c_{0}<c_{1}<\cdots<c_{n}<c_{n+1}:=\sup _{\mathbb{R}^{d}} u .
\end{gathered}
$$

Then, $\nabla u$ is isotropically realizable with a positive $C^{1}$-function $\sigma$ in the sets $\left\{c_{j}<u<c_{j+1}\right\}$ for $j=0, \ldots, n$.

\section{Example 2.2.}

1. Let $u: \mathbb{R}^{2} \rightarrow \mathbb{R}$ be the function defined by

$$
u(x):=\arctan \left(x_{1}\right)+\arctan \left(x_{2}\right) \quad \text { for } x=\left(x_{1}, x_{2}\right) \in \mathbb{R}^{2} .
$$

We have

$$
\forall x \in \mathbb{R}^{2}, \quad \frac{\partial_{x_{2}} u(x)}{\partial_{x_{1}} u(x)}=\frac{x_{1}^{2}+1}{x_{2}^{2}+1}
$$

such that condition (2.3) holds true with $\alpha_{1}(t)=\alpha_{2}(t)=\beta_{1}(t)=\beta_{2}(t)=t^{2}+1$.

Therefore, $\nabla u$ is isotropically realizable in $\mathbb{R}^{2}$ while $\inf _{\mathbb{R}^{2}}|\nabla u|=0$.

2. Let $u: \mathbb{R}^{3} \rightarrow \mathbb{R}$ be the function defined by

$$
u(x):=x_{1}^{3}+x_{2}^{3}+x_{3}^{3}+x_{1}^{2} x_{2}+x_{1} x_{2}^{2}+x_{1}^{2} x_{3}+x_{1} x_{3}^{2}+x_{2}^{2} x_{3}+x_{2} x_{3}^{2} \quad \text { for } x=\left(x_{1}, x_{2}, x_{3}\right) \in \mathbb{R}^{3} .
$$

We have

$$
\forall x \in \mathbb{R}^{3}, \quad\left\{\begin{array}{l}
\partial_{x_{1}} u(x)=3 x_{1}^{2}+x_{2}^{2}+x_{3}^{2}+2 x_{1} x_{2}+2 x_{1} x_{3} \\
\partial_{x_{2}} u(x)=x_{1}^{2}+3 x_{2}^{2}+x_{3}^{2}+2 x_{1} x_{2}+2 x_{2} x_{3} \\
\partial_{x_{3}} u(x)=x_{1}^{2}+x_{2}^{2}+3 x_{3}^{2}+2 x_{1} x_{3}+2 x_{2} x_{3} .
\end{array}\right.
$$

The partial derivatives of $u$ thus turn to be 3 quadratic forms on $\mathbb{R}^{3}$ associated with 3 symmetric matrices of $\mathbb{R}^{3 \times 3}$ the eigenvalues of which are $0<2-\sqrt{3}<1<2+\sqrt{3}$. Hence, the function $u$ has $(0,0,0)$ as unique critical point. Moreover, we deduce that for any $x \in \mathbb{R}^{3} \backslash\{(0,0,0)\}$,

$$
\frac{2-\sqrt{3}}{2+\sqrt{3}} \leq\left\{\begin{array}{l}
\frac{\partial_{x_{2}} u(x)}{\partial_{x_{1}} u(x)}=\frac{x_{1}^{2}+3 x_{2}^{2}+x_{3}^{2}+2 x_{1} x_{2}+2 x_{2} x_{3}}{3 x_{1}^{2}+x_{2}^{2}+x_{3}^{2}+2 x_{1} x_{2}+2 x_{1} x_{3}} \\
\frac{\partial_{x_{3}} u(x)}{\partial_{x_{2}} u(x)}=\frac{x_{1}^{2}+3 x_{2}^{2}+3 x_{3}^{2}+2 x_{1} x_{3}+2 x_{2} x_{3}}{x_{1}^{2}+3 x_{2}^{2}+x_{3}^{2}+2 x_{1} x_{2}+2 x_{2} x_{3}}
\end{array}\right\} \leq \frac{2+\sqrt{3}}{2-\sqrt{3}},
$$

such that condition (2.3) holds true with constant functions $\alpha_{1}, \alpha_{2}, \alpha_{3}, \beta_{1}, \beta_{2}, \beta_{3}$.

Therefore, $\nabla u$ is isotropically realizable by a positive continuous function in the open sets $\{u>0\}$ and $\{u<0\}$.

3. Let $f \in C^{3}(\mathbb{R})$ be an increasing function such that

$$
\left\{x \in \mathbb{R}: f^{\prime}(x)=0\right\}=\{0,1\} .
$$


Define the function $u \in C^{3}\left(\mathbb{R}^{3}\right)$ by

$$
u(x):=\frac{f\left(x_{1}+x_{2}+x_{3}\right)+f\left(x_{1}+2 x_{2}+x_{3}\right)+f\left(x_{1}+x_{2}+3 x_{3}\right)+f\left(x_{1}+4 x_{2}+5 x_{3}\right)}{4} \quad \text { for } x \in \mathbb{R}^{3} .
$$

Due to the non-negativity of $f^{\prime}$ it is easy to check that $\nabla u(x)=0$

$$
\begin{aligned}
& \Leftrightarrow f^{\prime}\left(x_{1}+x_{2}+x_{3}\right)=f^{\prime}\left(x_{1}+2 x_{2}+x_{3}\right)=f^{\prime}\left(x_{1}+x_{2}+3 x_{3}\right)=f^{\prime}\left(x_{1}+4 x_{2}+5 x_{3}\right)=0 \\
& \Leftrightarrow x_{1}+x_{2}+x_{3}, x_{1}+2 x_{2}+x_{3}, x_{1}+x_{2}+3 x_{3}, x_{1}+4 x_{2}+5 x_{3} \in\{0,1\} \\
& \Leftrightarrow x_{1} \in\{0,1\}, x_{2}=x_{3}=0 .
\end{aligned}
$$

It follows that $(0,0,0)$ and $(1,0,0)$ are the only critical points of $u$ with $u(0,0,0)=f(0)$ and $u(1,0,0)=f(1)>f(0)$. Moreover, we have for any $x \in \mathbb{R}^{3} \backslash\{(0,0,0),(1,0,0)\}$,

$$
\begin{aligned}
& 1 \leq \frac{\partial_{x_{2}} u(x)}{\partial_{x_{1}} u(x)}=\frac{f^{\prime}\left(x_{1}+x_{2}+x_{3}\right)+2 f^{\prime}\left(x_{1}+2 x_{2}+x_{3}\right)+f^{\prime}\left(x_{1}+x_{2}+3 x_{3}\right)+4 f^{\prime}\left(x_{1}+4 x_{2}+5 x_{3}\right)}{f^{\prime}\left(x_{1}+x_{2}+x_{3}\right)+f^{\prime}\left(x_{1}+2 x_{2}+x_{3}\right)+f^{\prime}\left(x_{1}+x_{2}+3 x_{3}\right)+f^{\prime}\left(x_{1}+4 x_{2}+5 x_{3}\right)} \leq 4 \\
& \frac{1}{4} \leq \frac{\partial_{x_{3}} u(x)}{\partial_{x_{2}} u(x)}=\frac{f^{\prime}\left(x_{1}+x_{2}+x_{3}\right)+f^{\prime}\left(x_{1}+2 x_{2}+x_{3}\right)+3 f^{\prime}\left(x_{1}+x_{2}+3 x_{3}\right)+5 f^{\prime}\left(x_{1}+4 x_{2}+5 x_{3}\right)}{f^{\prime}\left(x_{1}+x_{2}+x_{3}\right)+2 f^{\prime}\left(x_{1}+2 x_{2}+x_{3}\right)+f^{\prime}\left(x_{1}+x_{2}+3 x_{3}\right)+4 f^{\prime}\left(x_{1}+4 x_{2}+5 x_{3}\right)} \leq 5,
\end{aligned}
$$

such that condition (2.3) holds true with constant functions $\alpha_{1}, \alpha_{2}, \alpha_{3}, \beta_{1}, \beta_{2}, \beta_{3}$.

Therefore, $\nabla u$ is isotropically realizable by a positive continuous function in the open sets $\{u<f(0)\},\{f(0)<u<f(1)\},\{u>f(1)\}$.

Proof of Theorem 2.1. Let $u \in C^{2}\left(\mathbb{R}^{d}\right)$.

Proof of $i$ ). Fix $x \in \mathbb{R}^{d}$. Let $0 \in\left(\tau_{-}, \tau_{+}\right)$be the maximal interval on which the gradient flow $X(\cdot, x)$ is solution to equation (1.3) with $b=\nabla u$. The times $\tau_{-}$and $\tau_{+}$do depend on $x$, but their dependence is omitted for the sake of simplicity. Define the function $f$

$$
f(t):=u(X(t, x)) \text { for } t \in\left(\tau_{-}, \tau_{+}\right) .
$$

First, let us prove that the range of $f$ agrees with the interval $\left(\inf _{\mathbb{R}^{d}} u, \sup _{\mathbb{R}^{d}} u\right)$, i.e.

$$
\left\{f(t): t \in\left(\tau_{-}, \tau_{+}\right)\right\}=\left(\inf _{\mathbb{R}^{d}} u, \sup _{\mathbb{R}^{d}} u\right) .
$$

In [6] it is immediate that the range of $f$ is $\mathbb{R}$, since the derivative $f^{\prime}=|\nabla u(X(\cdot, x))|^{2}$ is defined over the whole interval $\mathbb{R}$ and is bounded from below by a positive constant. Here, the flow $X(\cdot, x)$ is only defined on the interval $\left(\tau_{-}, \tau_{+}\right)$, and we may have $\inf _{\mathbb{R}^{d}}|\nabla u|=0$. For the sake of simplicity we write $X(t)$ in place of $X(t, x)$ in the sequel.

Assume by contradiction that the flow $X(t)$ is bounded in the neighborhood of $\tau_{+}$. Then, $\tau_{+}=\infty$, otherwise $X^{\prime}(t)$ is bounded in the neighborhood of $\tau_{+}$and the flow $X(t)$ could be extended beyond $\tau_{+}$(see, e.g., [9, Section 17.4]). Then, the derivative $f^{\prime}(t)=|\nabla u(X(t))|^{2}$ is bounded from below by a positive constant in the neighborhood of $\infty$, which implies that $f(t)=u(X(t))$ tends to $\infty$ as $t \rightarrow \infty$, a contradiction. Therefore, there exists an increasing sequence $t_{n} \geq 0$ which tends to $\tau_{+}$such that $\left|X\left(t_{n}\right)\right|$ tends to $\infty$ as $n \rightarrow \infty$.

From now on, we assume that all the partial derivatives of $u$ are non-negative. The nonpositivity case of condition (2.1) is quite similar. Denote by $A_{k}$ (respectively $B_{k}$ ) a primitive of the function $\alpha_{k}$ (respectively $\beta_{k}$ ) in condition $(2.3)$. We have for any $k \in\{1, \ldots, d-1\}$,

$$
\left\{\begin{array}{l}
A_{k}\left(X_{k}\left(t_{n}\right)\right)-A_{k+1}\left(X_{k+1}\left(t_{n}\right)\right) \leq A_{k}\left(x_{k}\right)-A_{k+1}\left(x_{k+1}\right) \\
B_{k}\left(X_{k}\left(t_{n}\right)\right)-B_{k+1}\left(X_{k+1}\left(t_{n}\right)\right) \geq B_{k}\left(x_{k}\right)-B_{k+1}\left(x_{k+1}\right) .
\end{array}\right.
$$


Hence, by virtue of condition (2.2) the non-decreasing sequences $X_{k}\left(t_{n}\right)$ and $X_{k+1}\left(t_{n}\right)$ either are both bounded or both tend to $\infty$. This combined with $\left|X\left(t_{n}\right)\right| \rightarrow \infty$ thus implies that all the sequences $X_{k}\left(t_{n}\right)$ tend to $\infty$ as $n \rightarrow \infty$. As a consequence, since $u$ is separately non-decreasing, we get that for any $y \in \mathbb{R}^{d}$,

$$
f\left(t_{n}\right)=u\left(X\left(t_{n}\right)\right) \geq u(y) \text { for any large enough } n,
$$

which yields $\sup _{\left(\tau_{-}, \tau_{+}\right)} f=\sup _{\mathbb{R}^{d}} u$. Similarly, we deduce from $(2.7)$ that $\inf _{\left(\tau_{-}, \tau_{+}\right)} f=\inf _{\mathbb{R}^{d}} u$. Therefore, since $f$ is increasing, we obtain the desired equality (2.6).

Now, fix a constant $c_{u}$ in the interval $\left(\inf _{\mathbb{R}^{d}} u, \sup _{\mathbb{R}^{d}} u\right)$. Then, for any $x \in \mathbb{R}^{d}$, there exists a unique $\tau(x) \in\left(\tau_{-}, \tau_{+}\right)$such that

$$
f(\tau(x))=u(X(\tau(x), x))=c_{u} \in\left(\inf _{\mathbb{R}^{d}} u, \sup _{\mathbb{R}^{d}} u\right) .
$$

Note that by virtue of the $C^{2}$-regularity of $u$, the flow $X(t, x)$ is a $C^{1}$-function (see, e.g., [9, Chap. 17.6]) such that $\partial_{t} X$ is non-vanishing. Thus, the implicit functions theorem implies that $\tau$ belongs to $C^{1}\left(\mathbb{R}^{d}\right)$.

Then, the proof of the isotropic realizability of $\nabla u$ follows the same scheme that the proof of [6, Theorem 2.15] with the time $\tau(x)$. More precisely, by the semi-group property of the flow

$$
X(s, X(t, x))=X(s+t, x) \text { for any } s, t \text { close to } 0,
$$

combined with the uniqueness of $\tau(x)$ we have for any $x \in \mathbb{R}^{d}$,

$$
\tau(X(t, x))=\tau(x)-t \text { for any } t \text { close to } 0 .
$$

Then, the $C^{1}$-function $\sigma$ defined by

$$
\sigma(x):=\exp \left(\int_{0}^{\tau(x)} \Delta u(X(s, x)) d s\right) \text { for } x \in \mathbb{R}^{d}
$$

by (2.8) and (2.9) satisfies for any $t$ close to 0 ,

$$
\sigma(X(t, x))=\exp \left(\int_{0}^{\tau(x)-t} \Delta u(X(s+t, x)) d s\right)=\sigma(x) \exp \left(-\int_{0}^{t} \Delta u(X(s, x)) d s\right) .
$$

Hence, differentiating the former equality with respect to $t$ and taking $t=0$, we get that for any $x \in \mathbb{R}^{d}$,

$$
\nabla \sigma(x) \cdot \nabla u(x)=-\sigma(x) \Delta u(x) \quad \text { or equivalently } \operatorname{div}(\sigma \nabla u)(x)=0 .
$$

Therefore, $\nabla u$ is isotropically realizable in $\mathbb{R}^{d}$ with the positive function $\sigma \in C^{1}\left(\mathbb{R}^{d}\right)$.

Proof of $i i)$. Let $x \in \mathbb{R}^{d}$ be such that $u(x)>u\left(x^{0}\right)$. Let us prove that the range of the function $f$ defined by $(2.5)$ contains the interval $\left(u\left(x^{0}\right), \sup _{\mathbb{R}^{d}} u\right)$, i.e.

$$
\left(u\left(x^{0}\right), \sup _{\mathbb{R}^{d}} u\right) \subset\left\{f(t): t \in\left(\tau_{-}, \tau_{+}\right)\right\} .
$$

First, note that

$$
\forall t \in\left(\tau_{-}, \tau_{+}\right), \quad f^{\prime}(t)=|\nabla(X(t, x))|^{2}>0 .
$$

Indeed, if $f^{\prime}\left(t_{0}\right)=0$ for some $t_{0}$, then $X\left(t_{0}, x\right)=x^{0}=X\left(t_{0}, x^{0}\right)$. Hence, by the uniqueness of the Cauchy-Lipschitz theorem, $X(t, x)=x^{0}$ for any $t \in\left(\tau_{-}, \tau_{+}\right)$and $x=X(0, x)=x^{0}$, a contradiction. 
The inequality (2.14) combined with the first argument of case $i$ ) implies that the flow $X(t)$ is not bounded in the neighborhood of $\tau_{+}$. Thus, as in the case $i$ ) with (2.7) we get that $\sup _{\left(\tau_{-}, \tau_{+}\right)} f=\sup _{\mathbb{R}^{d}} u$. Moreover, if the flow $X(t)$ is not bounded in the neighborhood of $\tau_{-}$, then we obtain similarly that $\inf _{\left(\tau_{-}, \tau_{+}\right)} f=\inf _{\mathbb{R}^{d}} u$. In this case the range of $f$ thus agrees with $\left(\inf _{\mathbb{R}^{d}} u, \sup _{\mathbb{R}^{d}} u\right)$, which implies $(2.13)$.

It thus remains to study the case where the flow $X(t)$ is bounded in the neighborhood of $\tau_{-}$, which implies that $\tau_{-}=-\infty$. Moreover, the function $f^{\prime}$ is not bounded by below by a positive constant in the neighborhood of $-\infty$, otherwise $f(t)=u(X(t))$ tends to $-\infty$ as $t \rightarrow-\infty$. Hence, there exists a decreasing sequence $t_{n} \leq 0$ which tends to $-\infty$ such that $X\left(t_{n}\right)$ tends to some point $\bar{x}$ and $f^{\prime}\left(t_{n}\right)=\left|\nabla u\left(X\left(t_{n}\right)\right)\right|^{2}$ tends to 0 as $n \rightarrow \infty$. At the limit we get that $\nabla u(\bar{x})=0$, which implies that $\bar{x}=x^{0}$ and $\inf _{\left(-\infty, \tau_{+}\right)} f=u\left(x^{0}\right)$. Therefore, by the increase of $f$ we obtain that the range of $f$ is $\left(u\left(x^{0}\right), \sup _{\mathbb{R}^{d}} u\right)$, which establishes (2.13).

Fix a constant $c_{u}$ in $\left(u\left(x^{0}\right), \sup _{\mathbb{R}^{d}} u\right)$. Then, for any $x \in \mathbb{R}^{d}$ such that $u(x)>u\left(x^{0}\right)$, there exists by $(2.13)$ a unique time $\tau(x) \in\left(\tau_{-}, \tau_{+}\right)$such that

$$
f(\tau(x))=u(X(\tau(x), x))=c_{u} \in\left(u\left(x^{0}\right), \sup _{\mathbb{R}^{d}} u\right) .
$$

Finally, we prove the isotropic realizability of $\nabla u$ in the open set $\left\{u>u\left(x^{0}\right)\right\}$ following the argument between (2.9) and (2.12) with the time $\tau(x)$. The proof of the isotropic realizability of $\nabla u$ in the open set $\left\{u<u\left(x^{0}\right)\right\}$ is quite similar.

Proof of iii). Let $x \in \mathbb{R}^{d}$ be such that $c_{j}<u(x)<c_{j+1}$ for some $j=0, \ldots, n$. Repeating the arguments of $i$ ) and $i i$ ) we have the following alternative satisfied by the function $f$ defined by (2.5):

- $X(t)$ is not bounded in the neighborhood of $\tau_{+}\left(\operatorname{resp} . \tau_{-}\right)$, then $\sup _{\left(\tau_{-}, \tau_{+}\right)} f=\sup _{\mathbb{R}^{d}} u$ (resp. $\inf _{\left(\tau_{-}, \tau_{+}\right)} f=\inf _{\mathbb{R}^{d}} u$ ),

- $X(t)$ is bounded in the neighborhood of $\tau_{+}$(resp. $\left.\tau_{-}\right)$, then $\tau_{+}=\infty\left(\operatorname{resp} . \tau_{-}=-\infty\right)$, and $\sup _{\left(\tau_{-}, \tau_{+}\right)} f \geq c_{j+1}\left(\operatorname{resp} \inf _{\left(\tau_{-}, \tau_{+}\right)} f \leq c_{j}\right)$.

Contrary to case $i i$ ), here we have only an inequality since $c_{j+1}$ (respectively $c_{j}$ ) is the smallest (respectively largest) critical value which can be attained asymptotically by the function $f$.

Hence, we deduce that

$$
\left(c_{j}, c_{j+1}\right) \subset\left\{f(t): t \in\left(\tau_{-}, \tau_{+}\right)\right\} .
$$

Finally, we conclude as before by considering a constant $c_{u} \in\left(c_{j}, c_{j+1}\right)$, the time $\tau(x)$ such that $u(X(\tau(x), x))=c_{u}$, and the conductivity $\sigma$ defined by (2.10) in the open set $\left\{c_{j}<u<c_{j+1}\right\}$.

\subsection{Isotropic realizability of a vector field in $\mathbb{R}^{d}$}

In this section we consider the isotropic realizability of a vector field $b \in C^{1}\left(\mathbb{R}^{d}\right)^{d}$. Consider the flow associated with the vector field $b$ defined by (1.3). In the sequel, $0 \in\left(\tau_{-}(x), \tau_{+}(x)\right)$ denotes the maximal interval on which the solution $X(\cdot, x)$ to $(1.3)$ is defined.

In the spirit of the former proof we have the following extension of Theorem 2.1.

Theorem 2.3. Let $b \in C^{1}\left(\mathbb{R}^{d}\right)^{d}$ and let $I$ be a non-empty open interval of $\mathbb{R}$. Assume that there exists a function $u \in C^{1}\left(\mathbb{R}^{d}\right)$ such that for any $x \in\{u \in I\}$, the function $f_{x}:=u(X(\cdot, x))$ satisfies $f_{x}^{\prime}>0$ in $\left(\tau_{-}(x), \tau_{+}(x)\right)$ and

$$
\forall x \in\{u \in I\}, \quad I \subset\left\{f_{x}(t): t \in\left(\tau_{-}(x), \tau_{+}(x)\right)\right\}
$$


where $\{u \in I\}$ denotes the inverse image of I by $u$. Then, the vector field $b$ is isotropically realizable in the open set $\{u \in I\}$ with a positive function $\sigma \in C^{1}(\{u \in I\})$.

Proof of Theorem 2.3. Fix a constant $c_{I}$ in the interval $I$, and let $x \in\{u \in I\}$. By (2.16) and the increase of $f_{x}$, there exists a unique $\tau(x) \in\left(\tau_{-}(x), \tau_{+}(x)\right)$ such that

$$
u(X(\tau(x), x))=c_{I} .
$$

By the semi-group property (2.8) of the flow $X$ combined with the uniqueness of $\tau$ the equality (2.9) still holds true. Moreover, by the implicit functions theorem $\tau$ belongs to $C^{1}(\{u \in I\})$. Therefore, following (2.10), (2.11), (2.12) with $b$ instead of $\nabla u$, the $C^{1}$-function $\sigma$ defined by

$$
\sigma(x):=\exp \left(\int_{0}^{\tau(x)}(\operatorname{div} b)(X(s, x)) d s\right) \quad \text { for } x \in\{u \in I\}
$$

is solution to the equation $\operatorname{div}(\sigma b)=0$ in the open set $\{u \in I\}$.

Example 2.4. Consider the gradient field $b=\nabla v$ in $\mathbb{R}^{2}$ defined by

$$
v(x):=\frac{1}{3}\left(x_{1}^{3}+x_{2}^{3}\right) \quad \text { for } x \in \mathbb{R}^{2} .
$$

Then, the flow $X$ defined by (1.3) is given by

$$
X(t, x)=\left(\frac{x_{1}}{1-t x_{1}}, \frac{x_{2}}{1-t x_{2}}\right), t \in\left(\tau_{-}(x), \tau_{+}(x)\right)=\left\{\begin{array}{cl}
\left(-\infty, \frac{1}{\max \left(x_{1}, x_{2}\right)}\right) & \text { if } x_{1}>0, x_{2}>0 \\
\left(\frac{1}{\min \left(x_{1}, x_{2}\right)}, \infty\right) & \text { if } x_{1}<0, x_{2}<0 \\
\left(\frac{1}{x_{1}}, \frac{1}{x_{2}}\right) & \text { if } x_{1} x_{2}<0 \\
\left(\frac{1}{x_{1}}, \pm \infty\right) & \text { if } \mp x_{1}>0, x_{2}=0 \\
\left(\frac{1}{x_{2}}, \pm \infty\right) & \text { if } x_{1}=0, \mp x_{2}>0 \\
\mathbb{R} & \text { if } x=(0,0) .
\end{array}\right.
$$

It is clear that the function $v$ satisfies condition (2.1) but not condition (2.3).

Define the function $u$ by $u(x):=x_{1}+x_{2}$ for $x \in \mathbb{R}^{2}$. The function $f_{x}:=u(X(\cdot, x))$ satisfies for any $x \neq(0,0)$,

$$
\forall t \in\left(\tau_{-}(x), \tau_{+}(x)\right), \quad f_{x}^{\prime}(t)=(\nabla u \cdot \nabla v)(X(t, x))=\left(X_{1}(t, x)\right)^{2}+\left(X_{2}(t, x)\right)^{2}>0,
$$

and

$$
\left\{f_{x}(t): t \in\left(\tau_{-}(x), \tau_{+}(x)\right)\right\}=\left\{\begin{array}{cl}
(0, \infty) & \text { if } x_{1}>0, x_{2}>0 \\
(-\infty, 0) & \text { if } x_{1}<0, x_{2}<0 \\
\mathbb{R} & \text { if } x_{1} x_{2}<0 \\
(0, \pm \infty) & \text { if } \pm x_{1}>0, x_{2}=0 \\
(0, \pm \infty) & \text { if } x_{1}=0, \pm x_{2}>0 \\
\{0\} & \text { if } x=(0,0) .
\end{array}\right.
$$

Hence, the function $u$ satisfies the conditions of Theorem 2.3 with $I=(0, \infty)$ and $I=(-\infty, 0)$. Define $c_{I}:= \pm 1$ if $I:=(0, \pm \infty)$, and let $x \in I$. Moreover, it is easy to check that the solution $\tau(x)$ of $(2.17)$ is given by

$$
\tau(x)=\frac{x_{1}+x_{2}-2 x_{1} x_{2}-\sqrt{\left(x_{1}-x_{2}\right)^{2}+4 x_{1}^{2} x_{2}^{2}}}{2 x_{1} x_{2}} \text { for } x_{1}+x_{2} \neq 0 .
$$


Therefore, using formula (2.18) we obtain that the gradient field $b=\nabla v$ is isotropically realizable in the open set $\left\{x_{1}+x_{2} \neq 0\right\}$ with the conductivity $\sigma \in C^{1}\left(\left\{x_{1}+x_{2} \neq 0\right\}\right)$ defined by

$$
\sigma(x)=\frac{1}{\left(1-\tau(x) x_{1}\right)^{2}\left(1-\tau(x) x_{2}\right)^{2}} \quad \text { for } x_{1}+x_{2} \neq 0 .
$$

Note that $b=\nabla v$ is isotropically realizable in the open sets $\left\{x_{1}, x_{2}>0\right\}$ and $\left\{x_{1}, x_{2}<0\right\}$ with the simpler conductivity $x \mapsto\left(x_{1} x_{2}\right)^{-2}$. However, Theorem 2.3 here provides a suitable explicit conductivity in the two larger connected domains $\left\{x_{1}+x_{2}>0\right\}$ and $\left\{x_{1}+x_{2}<0\right\}$.

\section{Existence of a positive invariant measure in the torus under the gradient invertibility}

\subsection{Non-existence of a positive invariant measure}

In this section we will show that the isotropic realizability in $\mathbb{R}^{d}$ of Theorem 2.1 under the positivity assumptions (2.1) and (2.3) cannot be extended to the torus, namely the existence of a positive $Y_{d}$-periodic invariant measure. In particular, note that any vector field $b \in C_{\sharp}^{0}\left(Y_{d}\right)^{d}$ satisfies conditions (2.1) and (2.3) with $b$ in place of $\nabla u$, if there exists a constant $\alpha>0$ such that

$$
\forall k \in\{1, \ldots, d\}, \quad b_{k} \geq \alpha \text { in } Y_{d} \quad \text { or } \forall k \in\{1, \ldots, d\}, \quad b_{k} \leq-\alpha \text { in } Y_{d} .
$$

First, we have the following non-existence result if some component of $b$ changes sign.

Proposition 3.1. Let $b$ a periodic vector field in $L_{\sharp}^{\infty}\left(Y_{d}\right)^{d}$. Assume that for some $k=1, \ldots, d$, say $k=1$ without loss of generality, there exist two measurable subsets $A_{1}, B_{1}$ of $[0,1]$ with positive Lebesgue measure, such that

$$
\left\{\begin{array}{lll}
\forall x_{1} \in A_{1}, & b_{1}\left(x_{1}, x^{\prime}\right)>0 & \text { a.e. } x^{\prime} \in \mathbb{R}^{d-1} \\
\forall x_{1} \in B_{1}, & b_{1}\left(x_{1}, x^{\prime}\right)<0 & \text { a.e. } x^{\prime} \in \mathbb{R}^{d-1}
\end{array}\right.
$$

Then, the vector field $b$ has no positive invariant measure $\sigma \in L_{\sharp}^{\infty}\left(Y_{d}\right)$.

Proof of Proposition 3.1. Assume by contradiction that there exists some positive function $\sigma \in L_{\sharp}^{\infty}\left(Y_{d}\right)^{d}$ such that $\operatorname{div}(\sigma b)=0$ in $\mathbb{R}^{d}$, or equivalently in the torus sense

$$
\forall \varphi \in H_{\sharp}^{1}\left(Y_{d}\right), \quad \int_{Y_{d}} \sigma(x) b(x) \cdot \nabla \varphi(x) d x=0 .
$$

If the function $\varphi$ only depends on the variable $x_{1}$, the former equation leads us to

$$
\forall \varphi \in H_{\sharp}^{1}(0,1), \quad \int_{0}^{1}\left(\int_{Y_{d-1}} \sigma\left(x_{1}, x^{\prime}\right) b_{1}\left(x_{1}, x^{\prime}\right) d x^{\prime}\right) \varphi^{\prime}\left(x_{1}\right) d x=0,
$$

which implies the existence of a constant $c \in \mathbb{R}$ such that

$$
\int_{Y_{d-1}} \sigma\left(x_{1}, x^{\prime}\right) b_{1}\left(x_{1}, x^{\prime}\right) d x^{\prime}=c \quad \text { a.e. } x_{1} \in[0,1] .
$$


Then, by assumption (3.2) combined with the Fubini theorem we get that

$$
\left\{\begin{array}{l}
\int_{A_{1} \times Y_{d-1}} \underbrace{\sigma\left(x_{1}, x^{\prime}\right) b_{1}\left(x_{1}, x^{\prime}\right)}_{>0} d x=c\left|A_{1}\right|>0 \\
\int_{B_{1} \times Y_{d-1}} \underbrace{\sigma\left(x_{1}, x^{\prime}\right) b_{1}\left(x_{1}, x^{\prime}\right)}_{<0} d x=c\left|B_{1}\right|<0,
\end{array}\right.
$$

which yields a contradiction.

However, the positivity property (3.1) satisfied by a vector field $b$ is not sufficient to ensure the existence of a positive periodic invariant measure as shows the following example.

Example 3.2. Consider the $Y_{2}$-periodic continuous gradient field $b=\nabla u$ defined in $\mathbb{R}^{2}$ by

$$
u(x):=\alpha x_{1}+\alpha x_{2}+2 \cos \left(2 \pi x_{1}\right) \cos \left(2 \pi x_{2}\right) \text { for } x \in \mathbb{R}^{2}, \quad \text { where } \alpha>4 \pi .
$$

We have $\partial_{x_{k}} u \geq \alpha-4 \pi>0$ in $Y_{2}$ for $k=1,2$, such that the gradient field $b=\nabla u$ satisfies condition (3.1). On the other hand, make the change of function

$$
v(y):=u(x)=\cos \left(4 \pi y_{1}\right)+2 \alpha y_{2}+\cos \left(4 \pi y_{2}\right), \quad \text { where } \quad\left\{\begin{array}{l}
x_{1}=y_{1}+y_{2} \\
x_{2}=y_{2}-y_{1}
\end{array}\right.
$$

Then, the gradient field $\nabla_{y} v$ is still $Y_{2}$-periodic. Moreover, by virtue of Proposition $3.1 \nabla_{y} v$ has not a $Y_{2}$-periodic positive invariant measure, since $\partial_{y_{1}} v$ only depends on the variable $y_{1}$ and changes sign. Also note that the orthogonality of the change of variables $x=P y$, where $P P^{T}=2 I_{2}$, preserves the isotropy. Indeed, for any $\sigma \in L_{\sharp}^{\infty}\left(Y_{2}\right)$, and for any $\varphi \in C_{c}^{\infty}\left(\mathbb{R}^{2}\right)$ and $\psi(y):=\varphi(x)$, we have $\nabla_{y} \psi(y)=P^{T} \nabla_{x} \varphi(x)$, and

$$
\begin{aligned}
\int_{\mathbb{R}^{2}} \sigma(P y) \nabla_{y} v(y) \cdot \nabla_{y} \psi(y) d y & =\int_{\mathbb{R}^{2}} \sigma(x) P P^{T} \nabla u(x) \cdot \nabla \varphi(x)|\operatorname{det} P|^{-1} d x \\
& =\int_{\mathbb{R}^{2}} \sigma(x) \nabla u(x) \cdot \nabla \varphi(x) d x
\end{aligned}
$$

which implies that

$$
\operatorname{div}_{y}\left(\sigma(P y) \nabla_{y} v\right)=0 \text { in } \mathbb{R}^{2} \Leftrightarrow \operatorname{div}(\sigma \nabla u)=0 \text { in } \mathbb{R}^{2}
$$

where the positive function $y \mapsto \sigma(P y)$ belongs to $L_{\sharp}^{\infty}\left(Y_{2}\right)$. Hence, the gradient field $\nabla u$ cannot have a $Y_{2}$-periodic positive invariant measure since $\vec{\nabla}_{y} v$ has not one. Therefore, the $Y_{2}$-periodic gradient field $\nabla u$ satisfies condition (3.1), but has not a $Y_{2}$-periodic positive invariant measure.

\subsection{Criterium for the existence of a positive invariant measure}

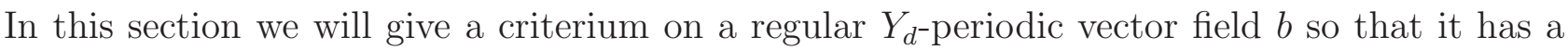
positive $Y_{d}$-periodic invariant measure. Let $b$ be a periodic vector field in $C_{\sharp}^{1}\left(Y_{d}\right)^{d}$, and consider the associated flow $X$ defined by (1.3).

We have the following result.

Theorem 3.3. Let $b \in C_{\sharp}^{0}\left(Y_{d}\right)^{d}$. Then, the following assertions are equivalent: 
i) There exist a positive function $\sigma \in C_{\sharp}^{0}\left(Y_{d}\right)^{d}$ and a vector field $V=\left(v_{1}, \ldots, v_{d}\right) \in C^{1}\left(\mathbb{R}^{d}\right)^{d}$ with $D V \in C_{\sharp}^{0}\left(Y_{d}\right)^{d \times d}$, such that

$$
\begin{gathered}
b \cdot \nabla v_{1}=1 \text { in } Y_{d}, \\
\sigma b=\left\{\begin{array}{ll}
R_{\perp} \nabla v_{2} & \text { if } d=2 \\
\nabla v_{2} \times \cdots \times \nabla v_{d} & \text { if } d \geq 3,
\end{array} \quad \text { in } Y_{d} .\right.
\end{gathered}
$$

ii) There exist a vector field $W \in C^{1}\left(\mathbb{R}^{d}\right)^{d}$ and a non-zero vector $\xi \in \mathbb{R}^{d}$ such that

$$
D W \in C_{\sharp}^{0}\left(Y_{d}\right)^{d \times d} \text { with }\langle D W\rangle=I_{d}, \quad \operatorname{det}(D W) \neq 0 \quad \text { in } Y_{d}, \quad(D W)^{T} b=\xi \text { in } Y_{d} .
$$

\section{Remark 3.4.}

1. The gradient invertibility (3.3) may seem rather sharp. But Proposition 3.5 below gives some general cases for which it holds true.

2. In dimension $d=2$ due to the representation of divergence free functions as orthogonal gradients, condition (3.4) is equivalent to the fact that $\sigma$ is a positive $Y_{2}$-periodic invariant measure. In higher dimension condition (3.4) only implies the existence of a positive $Y_{d}$-periodic invariant measure, since a divergence free vector field in $\mathbb{R}^{d}$ with $d \geq 3$, is not necessarily of the form (3.4).

\section{Proposition 3.5.}

i) Let $b \in C_{\sharp}^{0}\left(Y_{d}\right)^{d}$. Assume that

$$
\exists k \in\{1, \ldots, d\}, \quad b_{k}(x)=b_{k}\left(x_{k}\right)>0 \text { for } x \in \mathbb{R}^{d},
$$

then equality (3.3) holds true.

ii) Let $b \in C_{\sharp}^{1}\left(Y_{d}\right)^{d}$. Assume that for some $k \in\{1, \ldots, d\}$, there exists a function $u \in C^{1}\left(\mathbb{R}^{d}\right)$ such that $\nabla u$ is $Y_{d}$-periodic, $b \cdot \nabla u>0$ in $Y^{d}$, and the mapping

$$
\Phi:(t, x) \longmapsto \frac{D_{x} X(t, x) \nabla u(X(t, x))}{(b \cdot \nabla u)(X(t, x))} \text { is bounded and uniformly continuous in } \mathbb{R} \times \mathbb{R}^{d} \text {. }
$$

Then, condition (3.3) still holds true.

iii) Let $v_{1}, \ldots, v_{d}$ be $d \geq 2$ functions in $C^{1}\left(\mathbb{R}^{d}\right)$ such that

$$
\forall k \in\{1, \ldots, d\}, \quad \nabla v_{k} \text { is } Y_{d} \text {-periodic and }\left\|\nabla v_{k}-e_{k}\right\|_{L^{\infty}\left(Y_{d}\right)^{d}}<\varepsilon \text {. }
$$

Then, for any small enough $\varepsilon>0$, the vector field $b \in C_{\sharp}^{0}\left(Y_{d}\right)^{d}$ defined by

$$
\sigma:=\operatorname{det}\left(\nabla v_{1}, \nabla v_{2}, \ldots, \nabla v_{d}\right)>0 \quad \text { and } \quad \sigma b:= \begin{cases}R_{\perp} \nabla v_{2} & \text { if } d=2 \\ \nabla v_{2} \times \cdots \times \nabla v_{d} & \text { if } d>2\end{cases}
$$

satisfies condition (3.3).

\section{Remark 3.6.}

1. Condition (3.6) is a particular case of (3.7). Indeed, assuming (3.6) and choosing $u(x):=x_{k}$ we have

$$
X_{k}(t, x)=F^{-1}\left(t+F\left(x_{k}\right)\right) \quad \text { where } \quad F(t):=\int_{0}^{t} b_{k}^{-1}(s) d s .
$$


It follows that for any $(t, x) \in \mathbb{R} \times \mathbb{R}^{d}$,

$$
\frac{D_{x} X(t, x) \nabla u(X(t, x))}{(b \cdot \nabla u)(X(t, x))}=\frac{\nabla_{x} X_{k}(t, x)}{b_{k}(X(t, x))}=\frac{1}{b_{k}(X(t, x))} \frac{F^{\prime}\left(x_{k}\right) e_{k}}{F^{\prime}\left(X_{k}(t, x)\right)}=\frac{e_{k}}{b_{k}\left(x_{k}\right)},
$$

which clearly satisfies (3.7).

2. In condition (3.8) we may replace the canonical basis by any basis $\left(\xi^{1}, \ldots, \xi^{d}\right)$ of $\mathbb{R}^{d}$ such that $\operatorname{det}\left(\xi^{1}, \ldots, \xi^{d}\right)>0$.

Proof of Theorem 3.3. We prove the case $d \geq 3$. The case $d=2$ is quite similar.

i) $\Rightarrow$ ii) By (3.4) we have

$$
\sigma=\sigma b \cdot \nabla v_{1}=\operatorname{det}\left(\nabla v_{1}, \ldots, \nabla v_{d}\right)=\operatorname{det}(D V)
$$

which by the quasi-affinity of the cofactors (see, e.g., [8, Sec. 4.3.2]) implies that

$$
\operatorname{det}(\langle D V\rangle)=\langle\operatorname{det}(D V)\rangle=\langle\sigma\rangle>0 \text {. }
$$

Hence, the matrix $\langle D V\rangle$ is invertible, so that we may define the matrix $M$ of $\mathbb{R}^{d \times d}$ by

$$
M:=\langle D V\rangle^{-1}
$$

Let $W$ be the vector field defined by

$$
W:=M^{T} V \in C^{1}\left(\mathbb{R}_{d}\right)^{d},
$$

and let $\xi$ be the vector defined by

$$
\xi:=M^{T} e_{1} .
$$

Then, by (3.3), (3.4) and (3.14) we get that

$$
(D W)^{T} b=M^{T}(D V)^{T} b=M^{T}\left(\begin{array}{c}
b \cdot \nabla v_{1} \\
b \cdot \nabla v_{2} \\
\vdots \\
b \cdot \nabla v_{d}
\end{array}\right)=\xi
$$

Moreover, by (3.12) and (3.13) we have $\langle D W\rangle=I_{d}$, and by (3.11) we obtain that

$$
\operatorname{det}(D W)=\operatorname{det}(M) \operatorname{det}(D V)=\operatorname{det}(M) \sigma \neq 0 \quad \text { in } Y_{d} .
$$

Therefore, the function $W$ satisfies the desired condition (3.5).

$i i) \Rightarrow i$ ) Let $W$ be a vector field satisfying (3.5). Consider an invertible matrix $M \in \mathbb{R}^{d \times d}$ such that equation (3.14) holds true, and define the vector field $V$ by (3.13). Then, we have the equalities (3.15) which combined with (3.14) yield

$$
b \cdot \nabla v_{1}=1 \text { and } b \cdot \nabla v_{2}=\cdots=b \cdot \nabla v_{d}=0 \quad \text { in } Y_{d}
$$

which implies in particular (3.3). Moreover, we have

$$
\operatorname{det}(D W)=\operatorname{det}(M) \operatorname{det}(D V)=\operatorname{det}(M) \nabla v_{1} \cdot\left(\nabla v_{2} \times \cdots \times \nabla v_{d}\right) \neq 0 \quad \text { in } Y_{d} .
$$

Therefore, using a continuity argument and up to change $v_{2}$ in $-v_{2}$, the orthogonality conditions of (3.16) imply the existence of a positive function $\sigma \in C_{\sharp}^{0}\left(Y_{d}\right)$ such that condition (3.4) holds true, which concludes the proof. 


\section{Proof of Proposition 3.5.}

Proof of $i$ ). Assume that (3.6) holds true for some $k \in\{1, \ldots, d\}$, and let $v_{1}$ be the function defined by

$$
v_{1}(x):=\int_{0}^{x_{k}} b_{k}^{-1}(s) d s \quad \text { for } x \in \mathbb{R}^{d} .
$$

Therefore, $\nabla v_{1}=b_{k}^{-1} e_{k}$ is $Y_{d}$-periodic, and $b \cdot \nabla v_{1}=1$ in $Y_{d}$.

Proof of $i i)$. Let $x \in \mathbb{R}^{d}$. Define the function $f$ by $f(t):=u(X(t, x))$ for $t \in \mathbb{R}$. There exists a constant $c>0$ such that

$$
\forall t \in \mathbb{R}, \quad f^{\prime}(t)=(b \cdot \nabla u)(X(t, x)) \geq c,
$$

which implies that the range of $f$ is $\mathbb{R}$. Hence, there exists a unique $\tau(x) \in \mathbb{R}$ such that $f(\tau(x))=0$, and by the implicit functions theorem $\tau$ belongs to $C^{1}(\mathbb{R})$. Moreover, by the semi-group property of the flow combined with the uniqueness of $\tau(x)$, we have

$$
\forall t \in \mathbb{R}, \quad \tau(X(t, x))=\tau(x)-t .
$$

On the one hand, taking the derivative with respect to $t$ and choosing $t=0$, we get that

$$
b \cdot \nabla \tau=-1 \text { in } \mathbb{R}^{d} .
$$

On the other hand, differentiating with respect to $x$ the equality $u(X(\tau(x), x))=0$, we get that

$$
(b \cdot \nabla u)(X(\tau(x), x)) \nabla \tau(x)+D_{x} X(\tau(x), x) \nabla u(X(\tau(x), x))=0,
$$

or equivalently,

$$
\nabla \tau(x)=-\frac{D_{x} X(\tau(x), x) \nabla u(X(\tau(x), x))}{(b \cdot \nabla u)(X(\tau(x), x))}=-\Phi(\tau(x), x) .
$$

This combined with (3.7) implies that $\nabla \tau$ is bounded and uniformly continuous in $\mathbb{R}^{d}$. Hence, by the Ascoli theorem the average of gradient functions

$$
x \longmapsto \frac{-1}{(2 n+1)^{d}} \sum_{\kappa \in \mathbb{Z}^{d} \cap[-n, n]^{d}} \nabla \tau(x+\kappa)
$$

converges uniformly, up to a subsequence of $n$, to some continuous gradient $\nabla v_{1}$ in any compact set of $\mathbb{R}^{d}$. The function $\nabla v_{1}$ is clearly $Y_{d}$-periodic, and equality (3.17) implies (3.3).

Proof of iii). Condition (3.8) implies that

$$
\sigma=\operatorname{det}\left(e_{1}+\nabla v_{1}-e_{1}, \ldots, e_{d}+\nabla v_{d}-e_{d}\right)=1+O(\varepsilon),
$$

so that $\sigma$ is positive when $\varepsilon$ is small enough. Then, the vector field $b$ defined by (3.9) satisfies

$$
b \cdot \nabla v_{1}=\left\{\begin{array}{ll}
\sigma^{-1} \nabla v_{1} \cdot R_{\perp} \nabla v_{2} & \text { if } d=2 \\
\sigma^{-1} \nabla v_{1} \cdot\left(\nabla v_{2} \times \cdots \times \nabla v_{d}\right) & \text { if } d>2
\end{array}\right\}=\sigma^{-1} \operatorname{det}\left(\nabla v_{1}, \ldots, \nabla v_{d}\right)=1 \text { in } Y_{d},
$$

which concludes the proof. 


\section{Applications}

\subsection{Asymptotics of the flow}

There exists an interesting by-product of Theorem 3.3 in terms of the asymptotics of the flow $X$ defined by (1.3), which gives an alternative approach to the classical ergodic approach.

We have the following result.

Corollary 4.1. Let $b \in C_{\sharp}^{1}\left(Y_{d}\right)^{d}$ be a vector field such that conditions (3.3) and (3.4) hold true with functions $v_{k} \in C^{2}\left(\mathbb{R}^{d}\right)$. Then, there exists a vector $\xi \in \mathbb{R}^{d}$ such that the flow $X$ defined by (1.3) satisfies

$$
\forall x \in \mathbb{R}^{d}, \quad \lim _{|t| \rightarrow \infty} \frac{X(t, x)}{t}=\xi=\frac{\langle\sigma b\rangle}{\langle\sigma\rangle}
$$

Moreover, if there exists a non-zero vector $\lambda \in \mathbb{R}^{d}$ such that $b \cdot \lambda=0$ in $Y_{d}$, and if either $\sigma b$ is not constant in dimension $d=2$ or $\sigma b$ is not of the form $\lambda \times \nabla w$ in dimension $d=3$, then the flow $X$ is not ergodic.

\section{Remark 4.2.}

1. Condition (3.4) implies the existence of a positive $Y_{d}$-periodic invariant measure for the flow $X$ associated with the vector field $b \in C_{\sharp}^{1}\left(Y_{d}\right)^{d}$. Hence, by virtue of the Birkhoff ergodic theorem (see, e.g., [12, Chap. II, §5])

$$
b^{*}(x):=\lim _{|t| \rightarrow \infty} \frac{X(t, x)}{t} \text { exists for a.e. } x \in \mathbb{R}^{d},
$$

with respect to the Lebesgue measure, and $b^{*}$ is invariant by the flow $X$, i.e.

$$
b^{*}(X(t, x))=b^{*}(x) \quad \forall t \in \mathbb{R} \text {, a.e. } x \in \mathbb{R}^{d} .
$$

Under the extra assumption (3.3) Corollary 4.1 shows that limit (4.2) holds actually for every $x \in \mathbb{R}^{d}$. Moreover, the limit $b^{*}$ turns out to be the constant $\xi$ of (3.5), while the flow $X$ is not in general ergodic as shown in Example 4.3. Therefore, to prove (4.1) we need to use a different approach from the classical ergodic approach.

2. Formula (3.10) shows that in dimension $d=1$, under condition (3.3) or equivalently assuming that $b$ is a non-vanishing function in $C_{\sharp}^{1}(\mathbb{R})$, the flow $X$ is ergodic and asymptotics (4.1) holds with $\sigma=b^{-1}$ and the harmonic mean $\xi=\left\langle b^{-1}\right\rangle^{-1}$.

3. In the particular case of dimension two, assume that the vector field $b \in C_{\sharp}^{1}\left(Y_{2}\right)^{2}$ has a positive measure invariant $\sigma \in C_{\sharp}^{0}\left(Y_{2}\right)$ and does not vanish in $\mathbb{R}^{2}$. Then, using the Kolmogorov theorem (see [14, Lect. 11] or [16, Section 2]) Tassa [16, Section 3] obtained the following asymptotics

$$
\lim _{t \rightarrow \pm \infty} \frac{X(t, x)}{t}=a^{*}\left(e_{1}+\gamma e_{2}\right) \quad \text { for any } x \in \mathbb{R}^{d}, \quad \text { where } \gamma:=\frac{\left\langle\sigma b_{2}\right\rangle}{\left\langle\sigma b_{1}\right\rangle},
$$

with the alternative according to the so-called rotation number $\gamma$ :

- If $\gamma \notin \mathbb{Q}$, or equivalently the flow $X$ is ergodic, we have

$$
a^{*}=\frac{\left\langle\sigma b_{1}\right\rangle}{\langle\sigma\rangle} \quad \text { and } \quad a^{*}\left(e_{1}+\gamma e_{2}\right)=\frac{\langle\sigma b\rangle}{\langle\sigma\rangle} .
$$


- If $\gamma \in \mathbb{Q}$, we have in general

$$
a^{*}\left(e_{1}+\gamma e_{2}\right) \neq \frac{\langle\sigma b\rangle}{\langle\sigma\rangle} .
$$

In this case the gradient invertibility (3.3) cannot hold.

In view of the two points above, condition (3.3) gives the same asymptotics (4.1) than in the ergodicity setting, but does not imply the ergodicity of the flow. Moreover, the loss of condition (3.3) does not imply the loss of the ergodicity assumption. Therefore, condition (3.3) can be regarded as a substitute for the classical ergodicity assumption, since it induces a new and different regime for getting (4.1).

4. Peirone [11, Theorem 3.1] proved the asymptotics (4.1) everywhere in $\mathbb{R}^{2}$ under the sole condition that the vector field $b \in C_{\sharp}^{1}\left(Y_{2}\right)^{2}$ is non-vanishing in $\mathbb{R}^{2}$, using to this end the Birkhoff ergodic theorem combined with the Poincaré-Bendixson theorem (see, e.g., [9, Sec. 10.5]). Moreover, he provided [11, Lemma 4.6] an example of a non-vanishing vector field $b$ in $\mathbb{R}^{3}$ such that the asymptotics (4.1) does not hold at some point.

Therefore, Corollary 4.1 gives an alternative approach for proving (4.1) in dimension two in the absence of ergodicity assumption, and in higher dimension condition (3.4) gives a large class of vector fields $b$ such that (4.1) holds true everywhere in $\mathbb{R}^{d}$.

\section{Example 4.3.}

1. Let $v_{1} \in C^{1}(\mathbb{R})$ be a function such that $v_{1}^{\prime}$ is positive and 1-periodic, and let $v \in C^{1}(\mathbb{R})$ be a positive, 1-periodic and non-constant function. Define the vector field $b \in C_{\sharp}^{1}\left(Y_{2}\right)^{2}$ and the positive function $\sigma \in C_{\sharp}^{0}\left(Y_{2}\right)$ by

$$
b(x):=\frac{1}{v_{1}^{\prime}\left(x_{2}\right)} e_{2} \text { and } \sigma(x):=v\left(x_{1}\right) v_{1}^{\prime}\left(x_{2}\right) \quad \text { for } x \in \mathbb{R}^{2} .
$$

We have $b \cdot e_{1}=0$ in $Y_{2}, \sigma b=v\left(x_{1}\right) e_{2}$ is non-constant and divergence free, and condition (3.3) holds. Therefore, by virtue of Corollary 4.1 the flow $X$ defined by (1.3) is not ergodic and satisfies for any $x \in \mathbb{R}^{2}$,

$$
\lim _{|t| \rightarrow \infty} \frac{X(t, x)}{t}=\frac{\langle\sigma b\rangle}{\langle\sigma\rangle}=\frac{\left\langle v\left(x_{1}\right) e_{2}\right\rangle}{\left\langle v\left(x_{1}\right) v_{1}^{\prime}\left(x_{2}\right)\right\rangle}=\frac{e_{2}}{v_{1}(1)-v_{1}(0)} .
$$

2. Let $v_{1}, v_{2}, v_{3} \in C^{1}\left(\mathbb{R}^{3}\right)$ be 3 functions such that condition (3.8) holds true with small enough $\varepsilon>0$, and that $v_{2}(x)=v_{2}\left(x_{2}\right)$ has a 1-periodic and non-constant derivative. Then, define the positive function $\sigma \in C_{\sharp}^{0}\left(Y_{3}\right)$ and the vector field $b \in C_{\sharp}^{1}\left(Y_{3}\right)^{3}$ by

$\sigma:=\operatorname{det}\left(\nabla v_{1}, \nabla v_{2}, \nabla v_{3}\right)=\nabla v_{1} \cdot\left(\nabla v_{2} \times \nabla v_{3}\right)$ and $\sigma b=\nabla v_{2} \times \nabla v_{3}=v_{2}^{\prime}\left(x_{2}\right) e_{2} \times \nabla v_{3} \quad$ in $Y_{3}$,

so that $b \cdot \nabla v_{1}=1$ and $b \cdot e_{2}=0$ in $Y_{3}$.

On the other hand, for any $v \in C^{1}\left(\mathbb{R}^{3}\right)$ with $\nabla v Y_{3}$-periodic, the functions $\sigma b$ and $e_{2} \times \nabla v$ cannot agree. Otherwise, we have

$$
v_{2}^{\prime}\left(x_{2}\right)\left(\partial_{x_{3}} v_{3}(x) e_{1}-\partial_{x_{1}} v_{3}(x) e_{3}\right)=\partial_{x_{3}} v(x) e_{1}-\partial_{x_{1}} v(x) e_{3} \quad \text { for } x \in \mathbb{R}^{3},
$$

which implies that there exists a function $w \in C^{0}(\mathbb{R})$ such that

$$
v(x)=v_{2}^{\prime}\left(x_{2}\right) v_{3}(x)+w\left(x_{2}\right) \quad \text { for } x \in \mathbb{R}^{3} .
$$


Hence, by the $Y_{3}$-periodicity of $\nabla v_{3}$ and $\nabla v$ it follows that

$v\left(x_{1}, x_{2}, x_{3}+1\right)-v(x)=\left\langle\partial_{x_{3}} v\right\rangle=v_{2}^{\prime}\left(x_{2}\right)\left(v_{3}\left(x_{1}, x_{2}, x_{3}+1\right)-v_{3}(x)\right)=v_{2}^{\prime}\left(x_{2}\right)\left\langle\partial_{x_{3}} v_{3}\right\rangle \quad$ for $x \in \mathbb{R}^{3}$, a contradiction since $v_{2}^{\prime}$ is not constant and $\left\langle\partial_{x_{3}} v_{3}\right\rangle$ is close to 1 .

Therefore, by virtue of Corollary 4.1 the flow $X$ defined by (1.3) is not ergodic, and by the quasi-affinity of the cofactors satisfies for $x \in \mathbb{R}^{3}$,

$$
\lim _{|t| \rightarrow \infty} \frac{X(t, x)}{t}=\frac{\langle\sigma b\rangle}{\langle\sigma\rangle}=\frac{\left\langle\nabla v_{2}\right\rangle \times\left\langle\nabla v_{3}\right\rangle}{\left\langle\nabla v_{1}\right\rangle \cdot\left(\left\langle\nabla v_{2}\right\rangle \times\left\langle\nabla v_{3}\right\rangle\right)}=\frac{\left\langle\partial_{x_{3}} v_{3}\right\rangle e_{1}-\left\langle\partial_{x_{1}} v_{3}\right\rangle e_{3}}{\left\langle\partial_{x_{1}} v_{1}\right\rangle\left\langle\partial_{x_{3}} v_{3}\right\rangle-\left\langle\partial_{x_{3}} v_{1}\right\rangle\left\langle\partial_{x_{1}} v_{3}\right\rangle}
$$

Proof of Corollary 4.1. By virtue of Theorem 3.3 there exist a function $W \in C^{1}(\mathbb{R})^{d}$ and a non-zero vector $\xi \in \mathbb{R}^{d}$ satisfying (3.5). Define the function $W_{\sharp} \in C^{1}\left(\mathbb{R}_{d}\right)^{d}$ by

$$
W_{\sharp}(x):=x-W(x) \text { for } x \in \mathbb{R}^{d} .
$$

Note that the vector field $W_{\sharp}$ is $Y_{d^{-}}$periodic since $\langle D W\rangle=I_{d}$. Then, using that

$$
b=(D W)^{T} b+\left(D W_{\sharp}\right)^{T} b=\xi+\left(D W_{\sharp}\right)^{T} b,
$$

we have

$$
\begin{aligned}
X(t, x)=x+\int_{0}^{t} b(X(s, x)) d s & =x+t \xi+\int_{0}^{t}\left(\left(D W_{\sharp}\right)^{T} b\right)(X(s, x)) d s \\
& =x+t \xi+\int_{0}^{t} \frac{\partial}{\partial s}\left(W_{\sharp}(X(s, x))\right) d s \\
& =x+t \xi+W_{\sharp}(X(t, x))-W_{\sharp}(x) .
\end{aligned}
$$

Since the function $W_{\sharp} \in C_{\sharp}^{1}\left(Y_{d}\right)^{d}$ is bounded in $\mathbb{R}^{d}$, the former equality implies limit (4.1). Moreover, by (3.5) and the periodic div-curl lemma we have for any $\lambda \in \mathbb{R}^{d}$ and $w_{\lambda}:=W \lambda$,

$$
\langle\sigma\rangle \xi \cdot \lambda=\langle\sigma \xi \cdot \lambda\rangle=\left\langle\sigma b \cdot \nabla w_{\lambda}\right\rangle=\langle\sigma b\rangle \cdot\left\langle\nabla w_{\lambda}\right\rangle=\langle\sigma b\rangle \cdot \lambda
$$

which yields the second equality of (4.1).

Assume that there exists a non-zero vector $\lambda \in \mathbb{R}^{d}$ such that $b \cdot \lambda=0$ in $Y_{d}$, and that either $\sigma b$ is not constant in dimension $d=2$ or $\sigma b$ is not of the form $\lambda \times \nabla w$ in dimension $d=3$. Then, using the quasi-affinity of the determinant and (1.8) we have

$$
\operatorname{det}\left(\lambda,\left\langle\nabla v_{2}\right\rangle, \ldots,\left\langle\nabla v_{d}\right\rangle\right)=\left\langle\lambda \cdot\left(\nabla v_{2} \times \cdots \times \nabla v_{d}\right)\right\rangle=\langle\sigma b \cdot \lambda\rangle=0,
$$

which implies the existence of a non-zero vector $\left(\alpha_{1}, \alpha_{2}, \ldots, \alpha_{d}\right) \in \mathbb{R}^{d}$ such that

$$
\alpha_{1} \lambda+\alpha_{2}\left\langle\nabla v_{2}\right\rangle+\cdots+\alpha_{d}\left\langle\nabla v_{d}\right\rangle=0 .
$$

In view of (3.4) it follows that the function $v$ defined by

$$
v(x):=\alpha_{1} \lambda \cdot x+\alpha_{2} v_{2}(x)+\cdots+\alpha_{d} v_{d}(x) \text { for } x \in \mathbb{R}^{d},
$$

is in $C_{\sharp}^{1}\left(Y_{d}\right)$, and satisfies the equality $b \cdot \nabla v=0$ in $Y_{d}$, or equivalently (4.3) with $b^{*}=\nabla v$. Moreover, due to $\lambda \neq 0$ one of the coefficients $\alpha_{i}$ for some $i \geq 2$, is not null, say $\alpha_{2} \neq 0$ without loss of generality.

Now, assume that the function $v$ is constant. Then, when $d=2$ we have by (3.4)

$$
0=\nabla v=\alpha_{1} \lambda+\alpha_{2} \nabla v_{2}=\alpha_{1} \lambda-\alpha_{2} R_{\perp} \sigma b,
$$

a contradiction with the assumption that $\sigma b$ is not constant. When $d=3$ we have by (3.4)

$$
0=\nabla v \times \nabla v_{3}=\alpha_{1} \lambda \times \nabla v_{3}+\alpha_{2} \sigma b,
$$

again a contradiction with the assumption on $\sigma b$. Hence, the function $v$ is a non-constant invariant periodic function for the flow $X$. Therefore, the flow $X$ is not ergodic (see [12, Chap. II, §5]), which concludes the proof. 


\subsection{Homogenization of a linear transport equation}

For $b \in C_{\sharp}^{1}\left(Y_{d}\right)^{d}$ and $u_{0} \in C^{1}\left(\mathbb{R}^{d}\right)$, consider the following transport equation with an oscillating drift

$$
\begin{cases}\frac{\partial u_{\varepsilon}}{\partial t}-b(x / \varepsilon) \cdot \nabla u_{\varepsilon}=0 & \text { in }(0, \infty) \times \mathbb{R}^{d} \\ u_{\varepsilon}(0, x)=u_{0}(x) & \text { for } x \in \mathbb{R}^{d} .\end{cases}
$$

The homogenization of equation (4.6) was studied in the case of a two-dimensional divergence free vector field $b$ (i.e. with a constant invariant measure) through an ergodic approach by Brenier [3], then by Hou and Xin [10] with an oscillating initial datum which was specifically treated by a two-scale approach. Tassa [16] extended these results to any invariant measure in dimension two. These results show that the ergodicity of the flow associated with $b$ leads us to a homogenized linear transport equation. In contrast, the loss of ergodicity implies that the limit of $u_{\varepsilon}$ is not in general solution to a linear transport equation as Tartar [15] showed. Here, using the non-ergodic approach of Corollary 4.1 we obtain the following homogenization result in any dimension.

Corollary 4.4. Let $b \in C_{\sharp}^{1}\left(Y_{d}\right)^{d}$ be a vector field satisfying conditions (3.3) and (3.4) with functions $v_{k} \in C^{2}\left(\mathbb{R}^{d}\right)$, and let $u_{0} \in C^{1}\left(\mathbb{R}^{d}\right)$. Then, the solution $u_{\varepsilon}$ in $L_{\mathrm{loc}}^{1}\left(\mathbb{R}_{+} \times \mathbb{R}^{d}\right)$ to the transport equation (4.6) converges in $C_{\mathrm{loc}}^{0}\left(\mathbb{R}_{+} \times \mathbb{R}^{d}\right)$ to the function $u_{0}(x+t \xi)$ where the vector $\xi$ is given by (4.1).

Proof of Corollary 4.4. The flow $X_{\varepsilon}$ associated with the oscillating vector field $b(x / \varepsilon)$ is given by

$$
X_{\varepsilon}(t, x)=\varepsilon X(t / \varepsilon, x / \varepsilon) \quad \text { for }(t, x) \in \mathbb{R} \times \mathbb{R}^{d},
$$

where $X$ is the flow associated with $b$. Hence, due to the equality (4.5) and the boundedness of the function $W_{\sharp} \in C_{\sharp}^{1}\left(Y_{d}\right)^{d}$ defined by (4.4) it follows that

$$
X_{\varepsilon}(t, x)=x+t \xi+\varepsilon W_{\sharp}(X(t / \varepsilon, x / \varepsilon))-\varepsilon W_{\sharp}(x / \varepsilon)=x+t \xi+O(\varepsilon) .
$$

On the other hand, it is well known that the solution $u_{\varepsilon} \in L_{\text {loc }}^{1}\left(\mathbb{R}_{+} \times \mathbb{R}^{d}\right)$ to the transport equation (4.6) is given by

$$
u_{\varepsilon}(t, x)=u_{0}\left(X_{\varepsilon}(t, x)\right) \quad \text { for }(t, x) \in \mathbb{R}_{+} \times \mathbb{R}^{d} .
$$

Therefore, this combined with (4.8) and the continuity of $u_{0}$ implies that the sequence $u_{\varepsilon}$ converges uniformly to $u_{0}(x+t \xi)$ in any compact set of $\mathbb{R}_{+} \times \mathbb{R}^{d}$.

\section{References}

[1] G. Alessandrini: "An identification problem for an elliptic equation in two variables", Ann. Mat. Pura Appl., 145 (1986), 265-296.

[2] F. Bongiorno \& V. VAlente: "A method of characteristics for solving an underground water maps problem", Publ. I. A. C. III, 116 (1977).

[3] Y. BREnIER: "Remarks on some linear hyperbolic equations with oscillatory coefficients", Proceedings of the Third International Conference on Hyperbolic Problems (Uppsala 1990) Vol. I, II, Studentlitteratur, Lund (1991), 119-130. 
[4] M. Briane: "Isotropic realizability of electric fields around critical points", Disc. Cont. Dyn. Syst. B, 19 (2) (2014), 353-372.

[5] M. Briane: "Reconstruction of isotropic conductivities from non smooth electric fields", ESAIM: M2AN, 52 (3) (2018), 1173-1193.

[6] M. Briane, G.W. Milton \& A. Treibergs: "Which electric fields are realizable in conducting materials?", ESAIM: Math. Model. Numer. Anal., 48 (2) (2014), 307-323.

[7] I.P. Cornfeld, S.V. Fomin \& Ya.G. Sinai: Ergodic Theory, translated from the Russian by A.B. Sosinskii, Grundlehren der Mathematischen Wissenschaften [Fundamental Principles of Mathematical Sciences] 245, Springer-Verlag, New York, 1982, 486 pp.

[8] B. Dacorogna: Direct Methods in the Calculus of Variations, Applied Mathematical Sciences 78, Springer-Verlag, Berlin, 1989, 308 pp.

[9] M.W. Hirsch, S. Smale \& R.L. Devaney: Differential equations, Dynamical Systems, and an Introduction to Chaos, second edition, Pure and Applied Mathematics 60, Elsevier Academic Press, Amsterdam, 2004, 417 pp.

[10] T.Y. Hou \& X. XIN: "Homogenization of linear transport equations with oscillatory vector fields", SIAM J. Appl. Math., 52 (1) (1992), 34-45.

[11] R. Peirone: "Convergence of solutions of linear transport equations", Ergodic Theory Dynam. Systems, 23 (3) (2003), 919-933.

[12] M. Reed \& B. Simon: Methods of Modern Mathematical Physics. I. Functional analysis, revised and enlarged edition, Academic Press Inc., New York, 1980, 400 pp.

[13] G.R. Richter: "An inverse problem for the steady state diffusion equation", SIAM J. Appl. Math., 41 (2) (1981), 210-221.

[14] YA.G. SinAI: Introduction to Ergodic Theory, Translated by V. Scheffer, Mathematical Notes 18, Princeton University Press, Princeton, N.J., 1976, 144 pp.

[15] L. TARTAR: "Nonlocal effects induced by homogenization", Partial Differential Equations and the Calculus of Variations Vol. II, F. Colombini et al. (eds.), 925-938, Progr. Nonlinear Differential Equations Appl. 2, Birkhäuser Boston, Boston, MA, 1989.

[16] T. TAssA: "Homogenization of two-dimensional linear flows with integral invariance", SIAM J. Appl. Math., 57 (5) (1997), 1390-1405. 